\title{
Diagnóstico clínicopatológico do neuroma circunscrito solitário: relato de caso clínico
}

\author{
Clinical pathological diagnosis of solitary \\ circuncrytus neuroma: clinical case report
}

\author{
ANNE Diolina ARaÚJo MORAIS ${ }^{1}$ \\ MARCELO BONIFÁCIO DA SILVA SAMPIERI ${ }^{2}$ \\ FILIPE NOBRE CHAVES ${ }^{2}$ \\ Denise HÉlen Imaculada PEREIRA de OliVeIRA ${ }^{3}$
}

\begin{abstract}
RESUMO
Introdução: O neuroma circunscrito solitário, também denominado neuroma encapsulado em paliçada, é um tumor benigno neural, predominantemente cutâneo, constituído por quantidades variáveis de componentes de nervo periférico. Clinicamente, é descrito como uma pápula ou pequeno nódulo de superfície lisa, indolor e geralmente único. Na cavidade oral, a lesão é mais comum em palato duro.

Objetivo: O objetivo deste artigo é relatar um caso de neuroma circunscrito solitário focando nas características clínicas e histopatológicas, além de discutir os critérios morfológicos do diagnóstico diferencial com outras lesões derivadas da bainha nervosa periférica.

Relato de caso: O presente trabalho relata o caso de uma paciente do sexo feminino, 46 anos de idade, que se queixava de uma lesão assintomática na mucosa labial inferior com tempo de evolução de 2 anos. A lesão se mostrava como um nódulo de $5 \mathrm{~mm}$ de diâmetro, de cor semelhante à mucosa, superfície lisa, implantação séssil, consistência fibrosa e com um fator traumático associado, assim, tendo como hipótese diagnóstica elencada o fibroma traumático. Para tanto, foi realizada uma biópsia excisional e os cortes histológicos revelaram lóbulos de tecido mesenquimal contendo proliferação de células de núcleos ovoides e fusiformes, com cromatina frouxa e nucléolos inconspícuos. A reação imuno-histoquímica se exibiu positiva para proteína S-100 suportando o diagnóstico de neuroma circunscrito solitário.

Conclusão: O neuroma circunscrito solitário é pouco relatado na literatura e, por não se tratar de uma lesão comum, faz-se necessário um minucioso exame clínico e histopatológico com o auxílio de uma análise imuno-histoquímica para um diagnóstico preciso e correto. Além disso, é importante a realização de diagnósticos diferenciais com outras lesões.
\end{abstract}

Palavras-chave: Imuno-histoquímica. Neuroma. Diagnóstico.

\begin{abstract}
Introduction: The solitary circumscribed neuroma, also called palisade-encapsulated neuroma, is a benign neural tumor, predominantly cutaneous, consisting of varying amounts of peripheral nerve components. Clinically, it is described as a papule or small nodule with a smooth, painless and generally single surface. In the oral cavity, the lesion is more common on the hard palate.

Objective: The objective of this article is to report a case of a solitary circumscribed neuroma focusing on clinical and histopathological characteristics, in addition to discussing the morphological criteria of the differential diagnosis with other lesions derived from the peripheral nerve sheath.

Case report: The present study reports the case of a 46-year-old female patient who complained of an asymptomatic lesion on the lower lip mucosa with a 2-year evolution time. The lesion was presented as a nodule of $5 \mathrm{~mm}$ in diameter, similar in color to the mucosa, smooth surface, sessile implantation, fibrous consistency and with an associated traumatic factor, thus having the traumatic fibroma as a diagnostic hypothesis. For that, an excisional biopsy was performed and the histological sections revealed lobes of
\end{abstract}

\footnotetext{
${ }^{1}$ Acadêmico de Odontologia; Universidade Federal do Ceará Campus Sobral.

${ }^{2}$ Professor; Universidade Federal do Ceará Campus Sobral.

${ }^{3}$ Professor/a; Programa de Pós-graduação em Ciências da Saúde - Universidade Federal do Ceará Campus Sobral.
} 
mesenchymal tissue containing proliferation of ovoid and spindle cell cells, with loose chromatin and inconspicuous nucleoli. The immunohistochemical reaction is positive for S-100 protein supporting the diagnosis of solitary circumscribed neuroma.

Conclusion: Solitary circumscribed neuroma is rarely reported in the literature and, because it is not a common lesion, a thorough clinical and histopathological examination is necessary with the aid of an immunohistochemical analysis for a precise and correct diagnosis. In addition, it is important to make differential diagnoses with other injuries.

Keywords: Immunohistochemistry. Neuroma. Diagnosis.

\section{INTRODUÇÃO}

O neuroma encapsulado em paliçada (NEP) foi nomeado e descrito pela primeira vez por Reed e seus colaboradores, em 1972, em uma série de 44 casos. ${ }^{1} \mathrm{Em} 1989$, Fletcher discordou dessa nomeação e adotou uma terminologia alternativa para o mesmo, nomeouo de neuroma circunscrito solitário (NCS). ${ }^{2}$ Isto se deu pelo fato de o mesmo não apresentar características nucleares em paliçadas típicas, também observado por Dover et al. (1989), além de não ter encapsulamento total na maioria dos casos. ${ }^{2,3}$

O NCS é um tumor benigno neural, predominantemente cutâneo, constituído por quantidades variáveis de componentes de nervo periférico. ${ }^{1}$ Clinicamente, essa lesão é descrita como um pequeno nódulo de superfície lisa, indolor, de coloração semelhante à mucosa, geralmente único e sem fator causal comprovado. $1,4,5,6,7,8$ É mais comum na região de face, podendo ocorrer em outros lugares de forma mais rara, como na cavidade oral, preferencialmente na mucosa palatina. ${ }^{4,5} \mathrm{~A}$ lesão é com frequência diagnosticada entre a $5^{\mathrm{a}}$. e $7^{\mathrm{a}}$. década de vida e sem predileção por sexo. $4,9,10,11$ Além disso, as recidivas embora raras, já foram relatadas na literatura. ${ }^{9,6,12}$

Microscopicamente, o NCS é caracterizado por uma proliferação de células de Schwann e um número variável de axônios. ${ }^{4,5} \mathrm{Em}$ geral, uma fina cápsula envolve parcialmente a lesão. ${ }^{4,5,9,13} \mathrm{Em}$ relação aos marcadores imuno-histoquímicos, as células do NCS se mostram reativas à proteína
S-100. ${ }^{4,5,9}$ Além disso, as células da cápsula são positivas para antígeno de membrana epitelial (EMA $)^{4,5,9}$ e colágeno tipo IV. ${ }^{11}$ No entanto, ao contrário de outros tumores neurais, as células são negativas para proteína ácida fibrilar glial (GFAP). ${ }^{6}$

Os diagnósticos diferenciais mais associados ao NCS são fibroma traumático, carcinoma basocelular, papiloma e nevos. ${ }^{5,6,8,9}$ O tratamento de escolha para o NSC é a excisão cirúrgica local, ${ }^{6}$ podendo lançar mão de modalidades menos invasivas. ${ }^{7,13}$ Apesar de 0 NCS não ser considerado raro, poucos casos foram relatados na literatura.

O objetivo deste artigo é relatar um caso de NCS focando nas características clínicas e histopatológicas, além de discutir os critérios morfológicos do diagnóstico diferencial com outras lesões derivadas da bainha nervosa periférica.

\section{Relato de caso}

Paciente do sexo feminino, 46 anos de idade, procurou atendimento clínico se queixando de uma lesão assintomática na mucosa labial inferior com tempo de evolução de 2 anos. A lesão se apresentava como um nódulo de $5 \mathrm{~mm}$ de diâmetro, de cor semelhante à mucosa, superfície lisa, implantação séssil, consistência fibrosa e com fator traumático associado (Fig. 1A e 1B). De acordo com o relato da paciente, a mesma comentou que mordia involutariamente seu lábio diariamente. Logo, uma biópsia excisional foi realizada com a hipótese diagnóstica de fibroma traumático. 

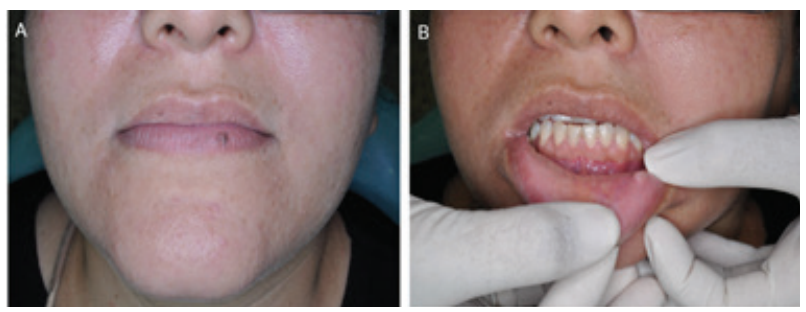

Figura 1: (A) Visão frontal; (B) Aspecto intraoral da lesão.

Microscopicamente, os cortes histológicos corados com hematoxilina e eosina revelaram lóbulos de tecido mesenquimal contendo proliferação de células de núcleos ovoides e fusiformes, com cromatina frouxa e nucléolos inconspícuos (Fig. 2A e 2B). Observou-se, também, vasos de pequeno calibre. A análise imuno-histoquímica revelou positividade para proteína S-100 suportando o diagnóstico de NCS (Fig. 2C e 2D).

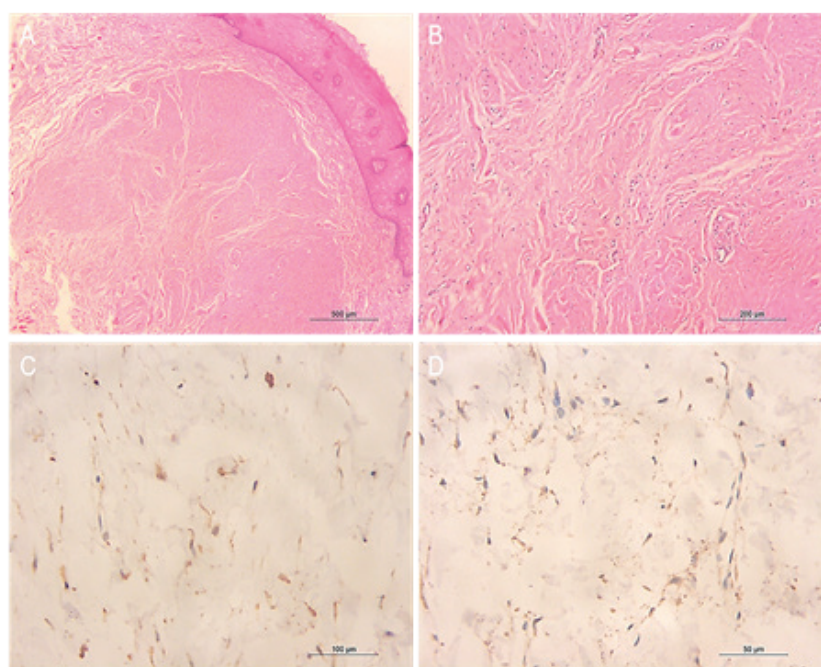

Figura 2: (A) NCS de padrão nodular exibindo proliferação de células consistentes com células de Schwann (slide view - $500 \mu \mathrm{m}$ ); (B) Células de Schwann organizadas em fascículos e microfacículos dispostos em várias direções e separados por tecido mixoide (slide view - $200 \mu \mathrm{m}$ ); (C) Marcação positiva para S-100 (slide view - $100 \mu \mathrm{m}$ ); (D) Detalhe da marcação citoplasmática do S-100 (slide view $-50 \mu \mathrm{m})$.

\section{Discussão}

O NCS é uma lesão benigna dos axônios e das células da bainha nervosa. ${ }^{14}$ Embora a maioria dos NCSs ocorra na pele, locais mucosos, como a glande do pênis, ${ }^{15}$ a fossa nasal $^{16}$ e a cavidade oral $^{4,5}$ também são afetadas, sendo as lesões orais o segundo local mais frequente, ${ }^{6}$ e tendo 0 palato como principal sítio de acometimento. ${ }^{4,5} \mathrm{O}$ lábio também pode ser citado como local de acometimento, inclusive se enquadrando na localização do presente caso. ${ }^{5,6,8}$ Além disso, clinicamente, o NCS caracteriza-se como um nódulo bem circunscrito, geralmente indolor que possui crescimento lento ao longo dos anos, ${ }^{6,9,17,18}$ como no caso atual, com tempo de evolução de aproximadamente dois anos e sem presença de sintomatologia associada.

As características clínicas dessa lesão foram bem mencionadas e discutidas nas séries de casos de Reed et al. (1972) e Fletcher (1989), porém, ambos, não concordavam em relação à nomenclatura. Independentemente da terminologia, NEP ou NCS, este é considerado um processo hiperplásico reativo, ${ }^{6,19}$ que pode estar associado a um trauma. ${ }^{3,4,20} \mathrm{Tal}$ associação corrobora o presente caso, o qual, de acordo com o relato da paciente, possuía um fator traumático envolvido.

Rotineiramente, o NCS tem um diagnóstico clínico variado, entre eles, os mais frequentes são nevos, fibroma traumático, papiloma e carcinoma basocelular. ${ }^{5,6,8,9}$ Esses diagnósticos diferenciais, também são os mais frequentes nos casos de NCS em região de lábio. ${ }^{5,8,9}$ No atual caso, pelo fato de a lesão se apresentar como uma lesão bem delimitada com superfície lisa de coloração semelhante à mucosa em região de lábio inferior, um diagnóstico clínico de fibroma traumático foi surgerido.

As características histopatológicas do NCS são descritas como uma proliferação de células muitas vezes em forma de fuso, consistentes com células de Schwann, acompanhadas por um número variável de axônios. ${ }^{6,7,8,9,19}$ Essa proliferação de células formam fascículos ou microfascículos entrelaçados, dispostos 
aleatoriamente. ${ }^{6,7}$ Ocasionalmente, esses fascículos podem ser separados por um tecido mixoide ou, por vezes, separados por fissuras artificiais. ${ }^{6,7,8,9,19}$ As células de Schwann mostram citoplasma eosinofílico e núcleos ondulados ou pontiagudos. 6,9 Embora os núcleos com frequência exibam uma orientação paralela dentro dos fascículos, um arranjo bem definido e em paliçadas e corpos de Verocay típicos do Schwannoma padrão Antoni $A$ não é geralmente visto. ${ }^{6,7,9,19}$ Pleomorfismo nuclear, atividade mitótica, fibrose e inflamação são raramente observados. ${ }^{6,7,19,21}$ A presença de cápsula, seja ela parcial ou total, é bem discutida atualmente. No decorrer dos casos, já documentados, observou-se que as lesões totalmente encapsuladas se encontravam em localizações mais profundas, logo, lesões mais superficiais dificilmente serão totalmente

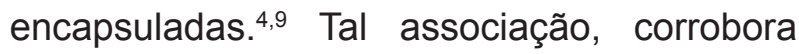
o atual caso, o qual aparecia de forma mais superficial e com cápsula pouco evidente.

Histopatologicamente, o padrão de crescimento nodular é o mais frequente, como observadonopresentecaso, mastambémforam relatados padrões tipos epitelioide, plexiforme e multinodular. ${ }^{6,8}$ Essas variações morfológicas não são clinicamente nem biologicamente relevantes, embora a familiaridade com elas minimize a confusão com outros tumores da bainha nervosa periférica, especialmente Schwannoma e neurofibroma. ${ }^{21}$

Em muitos casos, o tipo plexiforme é difícil de distinguir do multilobular. Porém, Koutlas \& Scheithauer (2010) usaram uma definição mais liberal, assim, definiram multilobular como lesões com lóbulos distintos, independentemente se sua disposição é perpendicular ou não. Além disso, afirmaram que esses padrões percebidos são altamente dependentes da forma de execução da técnica de processamento dos tecidos, assim, não estando relacionados com a biologia da lesão. ${ }^{6}$ $\mathrm{Na}$ verdade, a seção de etapas confirma a continuidade do que parecem nódulos individuais. ${ }^{2,20}$

Em relação aos diagnósticos diferenciais quanto aos aspectos histopatológicos, o NCS pode se assemelhar com alguns tumores da bainha nervosa periférica, como neuroma traumático, neurofibroma e Schawanoma, sendo os dois últimos os que apresentam maior relevância para o caso atual. No neuroma traumático, por associar-se a um trauma, a presença de células inflamatórias no tecido conjuntivo é bastante característica. ${ }^{18,22,23}$ No relato atual, a presença de um infiltrado inflamatório não foi digno de nota e nenhum sintoma associado foi relatado, como ocorre em casos de neuroma traumático. ${ }^{23}$

Em geral, o NCS pode ser diferenciado dos shwannomassemdificuldades. Doschwannoma, - NCS difere histopatologicamente por se localizar mais superficialmente em relação ao epitélio de superfície, além de não apresentar nenhum ou poucos axônios intralesionais e não exibir as células fusiformes dispostas em dois padrões distintos, Antoni A e B, e corpos de Verocay. ${ }^{6,18,24}$ De acordo com Koutlas \& Scheithauer (2010), o NCS, embora exiba os núcleos frequentemente com uma orientação paralela dentro dos fascículos, essa organização não se caracteriza como o padrão Antoni A do schwannoma. ${ }^{6}$

No neurofibroma, o padrão microscópico exibe células expostas em fascículos curtos ou espirais, ${ }^{25}$ de modo diferente do NCS, que mostra fascículos mais amplos e maior presença de axônios. ${ }^{18}$ Além disso, no neurofibroma, não há a presença de cápsula e, sim, de áreas hipocelulares, matriz mucoide (substância fundamental mucopolissacarídeo) com delicadas fibras de colágeno e mastócitos em quantidade significativa. , $^{6}$ Dessa forma, o arranjo das células neoplásicas do neurofibroma é a principal diferença microscópica do NCS. ${ }^{19}$ Além do mais, não há associação entre NCSs e neurofibromatose. ${ }^{6}$ 
Apesar das diferenças histopatológicas entre as lesões que fazem diagnóstico diferencial com o NCS, uma análise imuno-histoquímica pode-se fazer necessária para confirmação de diagnóstico. Entre os marcadores imunohistoquímicos, a proteína S-100 e colágeno IV exibem forte imunomarcação no citoplasma das células de Schwann do NCS. ${ }^{6,9,18}$ Além da S-100, é possível observar também imunorreatividade para a EMA nas células perineurais presentes na cápsula do NCS. ${ }^{4,6,9}$ Por sua vez, a GFAP exibe imunomarcação negativa nos casos de NCS. ${ }^{6,20,26}$

No neuroma traumático, a análise imunohistoquímica revela imunopositividade do S-100 nas células de schwann cercadas por células perineurais positivas para EMA, que contrastam com o NCS, na qual apenas a cápsula periférica contém células EMA+. ${ }^{6,21}$ Já para diferenciação do NCS com o neurofibroma, tem-se a presença de uma cápsula periférica negativa para EMA, onde o neurofibroma não exibe uma cápsula. ${ }^{4,6,21}$ No entanto, schwannomas e neurofibromas mostram coloração frequente para GFAP, de modo diferente do NCS que apresenta imunomarcação negativa. ${ }^{6,7,21}$

No presente caso, utilizou- se apenas o S-100 como marcador para distinção do NCS com o neurofibroma e schwannoma (Fig. 2C e 2D). O mesmo se mostrou positivo, assim, podendo concluir que a proliferação de células de núcleos ovoides e fusiformes, com cromatina frouxa e nucléolos inconspícuos, vista histopatologicamente, se tratava da proliferação das células de schwann, logo, confirmando e sustentando o diagnóstico de NCS. Tais diagnósticos diferenciais foram descartadados com base nas características histopatológicas, já discutidas, tendo o uso do S-100 como confirmação da proliferação de células de schwann características do NCS.

O tratamento convencional do NCS é a excisão cirúrgica tota, ${ }^{6}$ como realizada na paciente do caso relatado. Porém, atualmente, existem opções menos invasivas, como a criocirurgia e o uso de laser, que, além de maior conforto ao paciente, também proporciona uma melhor hemostasia. ${ }^{27}$ Apesar de não ser um tumor raro, as taxas de recorrência de NCS após tratamento são muito baixas. ${ }^{13} \mathrm{O}$ presente caso não mostrou recorrência em até 2 anos após o tratamento.

\section{CONSIDERAÇÕES FINAIS}

Embora o NCS seja uma lesão comum da bainha nervosa, é uma lesão pouco documentada, e isso levanta a questão a se pensar se o tumor é realmente não raro ou se é comumente subdiagnosticado, por se tratar de uma lesão assintomática. Assim, a análise histopatológica se torna primordial para obtenção do correto diagnóstico e, sempre que necessário, buscar métodos auxiliares, como os marcadores imuno-histoquímicos. Além disso, o conhecimento clínico é de extrema importância para se investigar os inúmeros diagnósticos diferenciais, confirmar o diagnóstico e realizar o tratamento adequado.

\section{REFERÊNCIAS}

1. Reed R., Fine R., Meltzer H. Palisaded encapsulated neuromas of the skin. Arch Dermatol. 1972; 106 (6): 865-70. https:// jamanetwork.com/journals/jamadermatology/ article-abstract/532949

2. Fletcher CD. Solitary circumscribed neuroma of the skin (so-called palisaded, encapsulated neuroma). A clinicopathologic and immunohistochemical study. Am J Surg Pathol. 1989; 13 (7): 574-80.

https://pubmed.ncbi.nlm.nih.gov/2660609/

3. Dover JS, From L., Lewis A. Palisaded encapsulated neuromas. Arch Dermatol. 1989; 125: 386-9.

https://jamanetwork.com/journals/ jamadermatology/article-abstract/550321 
4. Chauvin PJ, Wysocki GP, Daley TD, Pringle GA. Palisaded encapsulated neuroma of oral mucosa. Oral Surg Oral Med Oral Pathol. 1992, Jan.; 73 (1): 71-4. https://pubmed.ncbi. nlm.nih.gov/1603569/

5. Magnusson B. Palisaded encapsulated neuroma (solitary circumscribed neuroma) of the oral mucosa. Oral Surg Oral Med Oral Pathol Oral Radiol Endod. 1996; 82: 302-4.

https://www.oooojournal.net/article/S10792104(96)80356-8/pdf

6. Koutlas IG, Scheithauer BW. Palisaded encapsulated ("solitary circumscribed") neuroma of the oral cavity: a review of 55 cases. Head Neck Pathol. 2010; 4 (1): 15-26.

h t t p s : / / I i k . s p ri n g e r. c o m / article/10.1007\%2Fs12105-010-0162-x

7. Atarbashi-Moghadam S., Lotfi A., Zalani SS, Mokhtari S. Palisaded encapsulated (solitary circumscribed) neuroma of the buccal mucosa: a rare case. J Dent (Shiraz). 2017; 18: 314-17.

https://www.ncbi.nlm.nih.gov/pmc/articles/ PMC5702437/

8. Leblebici C., Savli TC, Yeni B., Cin M., Aksu AE. Palisaded encapsulated (solitary circumscribed) neuroma: a review of 30 cases. International Journal of Surgical Pathology. 2019: 1-9. https://journals.sagepub.com/doi/full/10.1177/ 1066896919833172?url_ver=Z39.882003\&rfr_ id $=$ ori\%3Arid\%3Acrossref.org\&rfr_dat $=\mathrm{cr}$ pub++0pubmed

9. Dakin MC, Leppard B, Theaker JM. The palisaded, encapsulated neuroma (solitary circumscribed neuroma). Histopathology. 1992; 20: 405-410. https://onlinelibrary. wiley.com/doi/abs/10.1111/j.13652559.1992. tb01010.x?sid=nlm\%3Apubmed

10. Jordan RC, Regezi JA. Oral spindle cell neoplasms: a review of 307 cases. Oral Surg Oral Med Oral Pathol Oral Radiol Endod. 2003; 95: 717-24.

https://www.oooojournal.net/article/S10792104(03)00058-1/fulltext

11. Manchanda AS, Narang RS, Puri G. Palisaded encapsulated neuroma. J Orofac Sci. 2015; 7: 136-139. doi: 10.4103 / 0975-8844.164308.

http://www.jofs.in/article.asp?issn=09758844; year=
2015; volume $=7 ;$ issue $=2 ;$ spage $=136 ;$ epage $=13$ 9; aulast=Manchanda;type $=3$

12. Lombardi T., Samson J., Kuffer R. Solitary circumscribed neuroma (palisaded encapsulated neuroma) of the oral mucosa. Ann Dermatol Venereol. 2002, Feb 129 (2): 229-32. https:// pubmed.ncbi.nlm.nih.gov/11937964/

13. Veerendranath RP. "Rare or underdiagnosed?" - solitary circumscribed neuroma of the lip. Journal of Clinical and Diagnostic Research. 2015, Jul 9 (7): 32-34. DOI: 10.7860/ JCDR/2015/14332.6255. https://www.ncbi.nlm. nih.gov/pmc/articles/PMC4573061/

14. Liu GY, Song H., Xu XL. Multiple palisaded encapsulated neuromas in siblings: a case report and review of the published work. J Dermatol. 2016; 43: 560-563. doi: 10.1111/13468138.13154. https://onlinelibrary.wiley.com/doi/ abs/10.1111/1346-8138.13154

15. Navarro M., Vilata J., Requena C., Aliaga A. Palisaded encapsulated neuroma (solitary circumscribed neuroma) of the glans penis. $\mathrm{Br}$ J Dermatol. 2000; 142: 1.061-2.

https://onlinelibrary.wiley.com/doi/abs/10.1046/j.13 652133.2000.03507.x?sid=nlm\%3Apubmed

16. Mayorga M., Acebo E., Val-Bernal JF. Palisaded encapsulated neuroma of the nasal fossa. Otolaryngol Head Neck Surg. 1998; 119: 141-3.

ht tps://journals.sagepub.com/doi/ full/10.1016/S01945998(98)701962?url_ ver $=Z 39.882003 \&$ rfr_id=ori:rid:crossref. org\&rfr_dat=cr_pub\%20\%200pubmed

17. Samdurkar A., Poudel S., Regmi S., Khurana S. Palisaded encapsulated neuroma (pen): a case report. J Univers Coll Med Sci. 2013; 1 (1): 28-32.

https://www.nepjol.info/index.php/JUCMS/article/ view/8420

18. Maharudrappa B., Kumar GS. Neural tumours of oral and para oral region. Int $\mathbf{J}$ dent $\mathbf{C l i n}$. 2011; 3 (1): 34-43.

https://www.semanticscholar.org/paper/NeuralTumours-of-Oral-and-Para-Oral-RegionMaharudrappa-Kumar/e8f0bec72a600149845e 31835258ed2bb0748535?p2df

19. Mortazavi N., Gholami A., Shakib PA, Hosseinkazemi H. Palisaded encapsulated 
neuroma of the tongue clinically mimicking a pyogenic granuloma: a case report and review of literature. Journal of Dentistry, Tehran University of Medical Sciences. 2015; 12 (7). https://www.ncbi.nlm.nih.gov/pmc/articles/ PMC4749420/

20. Albrecht S., Kahn HJ, From L. Palisaded encapsulatedneuroma:animmunohistochemical study. Mod Pathol. 1989; 2: 403-6. https:// europepmc.org/article/med/2503821

21. Rodríguez-Peralto JL, Riveiro-Falkenbach E., Carrillo R. Benign cutaneous neural tumors. Semin Diagn Pathol. 2013; 30: 45-57.

https://www.sciencedirect.com/science/article/abs/ pii/S0740257012000135?via\%3Dihub

22. Anbinder AL, Paiva TV, Mariano RC, Quirino MRS. Traumatic neuroma of the lower lip. West Indian Med J. 2009; 58 (6): 604-06. https:// pubmed.ncbi.nlm.nih.gov/20583693/

23. Kerr AR, Phaelan JA. Benign lesions of the oral cavity, 11. ed. Burket's Oral Medicine; 2008: 133-4.

24. Folpe AL. Soft tissue tumors of the head and neck. Diagnostic Surgical Pathology of the Head and Neck. 2009: 670-1. ht t ps://www.researchgate.net/ publication/283375630_SoftTissue_Tumors_ of_the_Head_and_Neck

25. Lopes MA, Vargas PA, Almeida OP, Takahama A., León JE. Oral traumatic neuroma with mature ganglion cells: A case report and review of the literature. J Oral Maxillofac Pathol. 2009; 13: 67-69.

http://www.jomfp.in/article.asp?issn=0973029X;yea $r=2009$; volume $=13$;issue $=2$; spage $=67$; epage $=$ 69;aulast=Lopes

26. Argenyi ZB. Immunohistochemical characterization of palisaded, encapsulated neuroma. J Cutan Pathol. 1990; 17: 329-35.

https://onlinelibrary.wiley.com/doi/ $\mathrm{abs} / 10.1111 / \mathrm{j} .16000560 .1990$. tb00108.x?sid=nlm\%3Apubmed

27. Capodiferro S., Maiorano E., Scarpelli F., Favia G. Fibrolipoma of the lip treated by diode laser surgery: a case report. J Med Case Reports. 2008; 2: 301. doi: 10.1186 / 1752-1947-2301. https://www.ncbi.nlm.nih.gov/pmc/articles/ PMC2547114/

Submetido em: 27-6-2020

Aceito em: 24-9-2020 